\title{
1 Comparison of brain activity while tasting of passion fruit juice 2 sweetened with caloric and non-caloric sweeteners.
}

3

Discrimination of different sweeteners

5

6 Gustavo Voltani von Atzingen ${ }^{1}$, Hubert Luzdemio Arteaga. Miñano ${ }^{2}$, Amanda

7 Rodrigues da Silva ${ }^{1}$, Nathalia Fontanari Ortega ${ }^{3}$, Ernane Jose Xavier Costa ${ }^{3}$, Ana

8 Carolina de Sousa Silva ${ }^{3, *}$

$9{ }^{1}$ Instituto Federal de São Paulo, Piracicaba, 13414-155, Brasil;

$10^{2}$ Escuela Ingeniería Agroindustrial, Facultad de Ciencias Agrarias, Campus Colpa

11 Huacariz, National Autonomous Universidad Nacional Autonóma de Chota, Chota

12 06121, Peru

$13^{3}$ Departamento de Ciências Básicas, Faculdade de Zootecnia e Engenharia de Alimentos,

14 Universidade de São Paulo, 13635-900, São Paulo, Brasil

$15{ }^{*}$ Corresponding author anacss@usp.br; Tel.: +551935654255

16 


\section{Abstract}

18 Sweetener type can influence sensory properties and consumer's acceptance and

19 preference for low-calorie products. An ideal sweetener does not exist, and each

20 sweetener must be used in situations to which it is best suited. Aspartame and sucralose

21 can be good substitutes for sucrose in passion fruit juice. Despite the interest in artificial sweeteners, little is known about how artificial sweeteners are processed in the human brain. Here, we evaluated brain signals of 11 healthy sub-jects when they tasted passion fruit juice equivalently sweetened with sucrose $(9.4 \mathrm{~g} / 100 \mathrm{~g})$, sucralose $(0.01593 \mathrm{~g} / 100$ $\mathrm{g})$, or aspartame $(0.05477 \mathrm{~g} / 100 \mathrm{~g})$. Electroencephalograms were recorded for two sites in the gustatory cortex (i.e., C3 and C4). Data with artifacts were disregarded, and the artifact-free data were used to feed a CNN. Our results indicated that the brain responses distinguish juice sweetened with sucrose from juice sweetened with aspartame and sucralose.

30 Keywords: sucrose, sucralose, aspartame, electroencephalography (EEG),

31 convolutional neural network (CNN).

\section{Introduction}

33 Sugar has been the main sweetener in human diet for centuries. It represents a high 34 percentage of the human daily energy consumption, but it offers little additional nutritional value (Bassoli and Merlini, 2003; Caballero, 2013; Zorn et al., 2014).

36 Given that replacing sugar with non-caloric (or low-calorie) sweeteners have become

37 popular among consumers seeking to lose or to maintain weight. Moreover, foods with

38 the same sweetening capacity might be perceived differently due to their caloric content

39 (Frank et al., 2008; Hill et al., 2014). Additionally, the brain might respond distinctly to 40 perceptually similar and identical tastes (Andersen et al., 2019). 
41 A satisfactory method for collecting an artifact-free EEG signal has not been developed

42 yet, but there are important results relating gustatory stimuli to evoked potential parameters in humans (Andersen et al., 2019; Hashida et al., 2005; Jacquin-Piques et al., 2016; Linforth, 2000; Mouillot et al., 2020; Ohla et al., 2012). In addition, from the food science standpoint, several aspects must be considered when it comes to taste perception, including the hypothesis that the taste perception threshold is related not only to the sensitivity of the sensory organ — in this case, the tongue — but also to a cognitive process in the brain (Huang et al., 2006; Naim et al., 2002; Okamoto and Dan, 2007).

Any study involving equi-intense sweeteners (i.e., sweeteners used in equivalent amounts) must consider how sweeteners influence sensory properties and consumer's acceptance and preference for low-calorie products (Pinheiro et al., 2005). An ideal sweetener does not exist, so each sweetener is appropriate for specific situations

54 (Nabors, 2002). Passion fruit is a popular tropical fruit that has an important commercial variety named yellow passion fruit, which is used to prepare juice that requires sweetening (Deliza et al., 2005). Aspartame and sucralose can be good substitutes for sucrose in passion fruit juice (Rocha and Bolini, 2015b, 2015a). For this study, we have hypothesized that EEG can distinguish the stimuli resulting

59 from the consumption of drinks sweetened with caloric or non-caloric sweeteners. To

60 test this hypothesis, we have compared brain signals acquired in response to the

61 consumption of passion fruit juice sweetened with sucrose (caloric sweetener),

62 sucralose, or aspartame (low-calorie sweeteners).

\section{Materials and Methods}


64 The methods used herein were divided into three main sections: (1) Participant

65

66

67

68

69

70

selection, (2) acquisition of EEG signal from the selected group, and (3) signal processing and CNN (Convolutional Neural Network) training and tests. Our aim was to use a CNN to determine differences between stimuli.

\section{Stimuli}

The passion fruit juices were prepared in the laboratory; unsweetened passion fruit pulp (DeMarchi ${ }^{\mathrm{TM}}$ ) was employed. The following ratio was used: one part of pulp to two parts of water. The juice samples were sweetened with sucrose (União $\left.{ }^{\mathrm{TM}}\right)$, aspartame (Ajinomoto $^{\mathrm{TM}}$ ), or sucralose. Pure water was considered as reference. The samples were prepared one day before the experiment, stored at $4-6{ }^{\circ} \mathrm{C}$, and tested at room temperature.

The sweetener concentration in the passion fruit juice was chosen according to Rocha and Bolini's study (Rocha and Bolini, 2015b, 2015a), who stated that 9.4/100 g was an ideal sucrose concentration according to consumer's acceptance. They also stated that the equivalent aspartame and sucralose concentrations in passion fruit juice were 0.0547 $\mathrm{g} / 100 \mathrm{~g}$ and $0.0159 \mathrm{~g} / 100 \mathrm{~g}$, respectively.

\section{Participant Selection}

A total of 105 volunteers were included in this study. The volunteers comprised students, teachers, and employees aged 19-55 years, recruited on campus. They did not have diabetes, smoke, or use medications that affect taste or cognitive processes.

Preference was given to volunteers that were used to consuming passion fruit juice (or that at least had no aversion to the fruit taste).

Each volunteer received samples containing $30 \mathrm{~mL}$ of passion fruit juice sweetened with different amounts of sugar (i.e., 4.7, 7.05, 9.4, 11.75, and $14.1 \mathrm{~g}$ ). The samples 
were placed in disposable cups and numbered randomly between 0000 and 9999 (e.g.,

A7932). The participants received samples in a randomized sequence of concentrations

91 juice?" Answers were collected in a 9.0-cm visual scale (VAS). VASs are input

92 mechanisms that allow users to specify a value within a predefined range. The

93 volunteers were instructed to consider the centre of the scale as ideal sweetness, 0 as

94 less sweet than ideal, and 9 as sweeter than ideal. Our objective was to select

individuals with a perception of sugar ideal sweetness as close as possible to $9.4 \mathrm{~g} / 100 \mathrm{~g}$

and, among these individuals, to select those that had good ability to order the samples

97 according to the sugar concentration.

98 The volunteers were informed about the nature and aims of the experiments and

99 provided informed consent. The study was approved by the Ethics Committee of the

100 Animal Science and Food Engineering College (FZEA) of the University of São Paulo

101 (USP) (CAAE 59017516.6.0000.5422).

\section{EEG recording}

103 EEG signals were acquired from 11 individuals (both sexes) while they were tasting

104 passion fruit juice. The individuals had been selected according to item 'Participant

105 selection' and had signed an informed consent form (ICF).

106 EEG was carried out non-invasively on the scalp surface; the participants wore an EEG

107 cap. The signal was acquired at positions C3 and C4 in the primary gustatory cortex

108 (Hashida et al., 2005; Kobayakawa, 1999) as defined by the international 10-20

109 electrode disposal system (Jasper and H., 1958). This electrode position was chosen on

110 the basis of the study by Kabayakawa et al. (1999), who showed magnetic fields

111 recorded from the brain (i.e., MEG) in response to two tastants: $1 \mathrm{M} \mathrm{NaCl}$ and $3 \mathrm{mM}$ 
112 saccharin, one of which was more activated in the central area of the head at positions

$113 \mathrm{C} 3$ and $\mathrm{C} 4$. The ground electrodes were placed on the participant's ear lobes, and a

114 reference electrode was placed on the forehead. The signals were sampled at $512 \mathrm{~Hz}$ by

115 using an iCelera digital portable electroencephalograph. Each recording lasted 16

116 seconds.

117 The volunteers were accommodated inside a Faraday cage, and the EEG signal started

118 being recorded when the volunteer drank the solution (juice). The volunteers were

119 instructed to keep their eyes closed and not to move during the recordings. Each

120 volunteer received eight $30-\mathrm{mL}$ samples for evaluation. The samples were placed in

121 disposable cups and numbered randomly between 0000 and 9999 (e.g., A7932). The

122 samples were offered in duplicate in the following order: water (reference), passion fruit

123 juice sweetened with sucrose, passion fruit juice sweetened with sucralose, and passion

124 fruit juice sweetened with aspartame. Each volunteer participated on three different

125 days of the experiment (repetitions). In the intervals between the sweetened samples,

126 the participants received sparkling water to clean residues from their palate and to

127 reduce other interferences.

128 Signal processing and CNN training

129 The signal was processed by using the Python programming language and the Pandas,

130 NumPy, and SciPy libraries. These libraries are employed to solve mathematical and

131 scientific problems, including signal processing. Initially, the data were inspected, and

132 the recordings with many artifacts were excluded from the database. The remaining data

133 consisted of signals from 68 experiments lasting 16 seconds each, recorded in two

134 channels (i.e., C3 and C4). These signals came from one of four stimuli (i.e., sucrose,

135 sucralose, aspartame, or water) and were captured across three days of repetitions per 
136 volunteer. The data were bandpass-filtered from 8 to $40 \mathrm{~Hz}$, and each signal was

137 divided into two-second segments with a 0.1 second stride. This means that 16 -second

138 segments (512 samples per second) were resampled in 9520 vectors at a length of 1028.

139 The EEG dataset and the processing code that supported the findings of this study are

140 available on GitHub (https://github.com/Atzingen/EEG Sweetners.).

141 When the vectors with length 1028 were used as inputs for the $\mathrm{CNN}$, they were

142 structured as a network consisting of three parallel convolution processes merged into a

143 fully two-layer-deep connected network.

144 The deep learning model was built by using an open-source Python library, i.e.,

145 Keras, which runs in a TensorFlow background. Optimal performance (e.g., number of

146 neurons on a given layer and number of filters and kernel size on the convolution layer)

147 was determined by using manual fine adjustment of the hyperparameters (e.g., number

148 of neurons per fully connected layer and number of filters and kernel size for

149 convolutional layers).

150 The last network layer contained four neurons, which were responsible for

151 mapping four possible results of an entry (water, sugar, aspartame, or sucralose). A

152 categorical cross-entropy loss function was used.

153 Training was accomplished by using an Adam algorithm with two-thirds of the

154 data, while the test was performed with the remaining one-third.

155 Finally, performance evaluation metrics, such as accuracy, precision, recall and

156 F1 score were calculated as follows.

$$
\begin{array}{r}
\text { accuracy }=\frac{T P+T N}{T P+T+F P+F N} \\
\text { precision }=\frac{T P}{T P+F P}
\end{array}
$$




$$
\text { recall }=\frac{T P}{T P+F N}
$$

$$
F 1 \text { score }=\frac{2(\text { precision }) *(\text { recall })}{(\text { precision })+(\text { recal })}
$$

162 where TP (true positive), FP (false positive), TN (true negative) and FN (false negative)

163 are technical terms for binary classifier. Specific, TP is the positive samples correctly

164 classified, FP is the positive samples misclassified, TN is the negative samples correctly

165 classified and FN represents the negative samples misclassified.

\section{Results and Discussion}

\section{Participant selection}

168 We selected the groups according to two criteria, namely, preference for the sample

169 with a sucrose concentration of $9.4 \mathrm{~g} / 100 \mathrm{~g}$ and good ability to order the sample

170 concentrations. This means that the selected participants indicated values around $4.5 \mathrm{~cm}$

171 on the VAS, which was equivalent to a sucrose concentration of $9.4 \mathrm{~g} / 100 \mathrm{~g}$.

172 Furthermore, they were able to order all the concentrations properly. Of the individuals

173 considered fit, 11 agreed to participate in the brain signal acquisition stage.

174 Figure 1-a illustrates the scale presented to the participants. Figure 1-b shows

175 participant A, who placed all the samples in the correct sequence of concentrations and

176 chose the sample with sugar concentration of $9.4 \mathrm{~g} / 100 \mathrm{~g}$ as his favourite. He was

177 selected for the next step. Participants B and C were not selected. Participant B (Figure

178 1-c) placed the samples correctly, but his preferred sample was not the sample with

179 sugar concentration of $9.4 \mathrm{~g} / 100 \mathrm{~g}$. Although participant C (Figure 1-d) preferred the

180 sample with a sugar concentration of $9.4 \mathrm{~g} / 100 \mathrm{~g}$, he was not able to order the samples

181 correctly. 


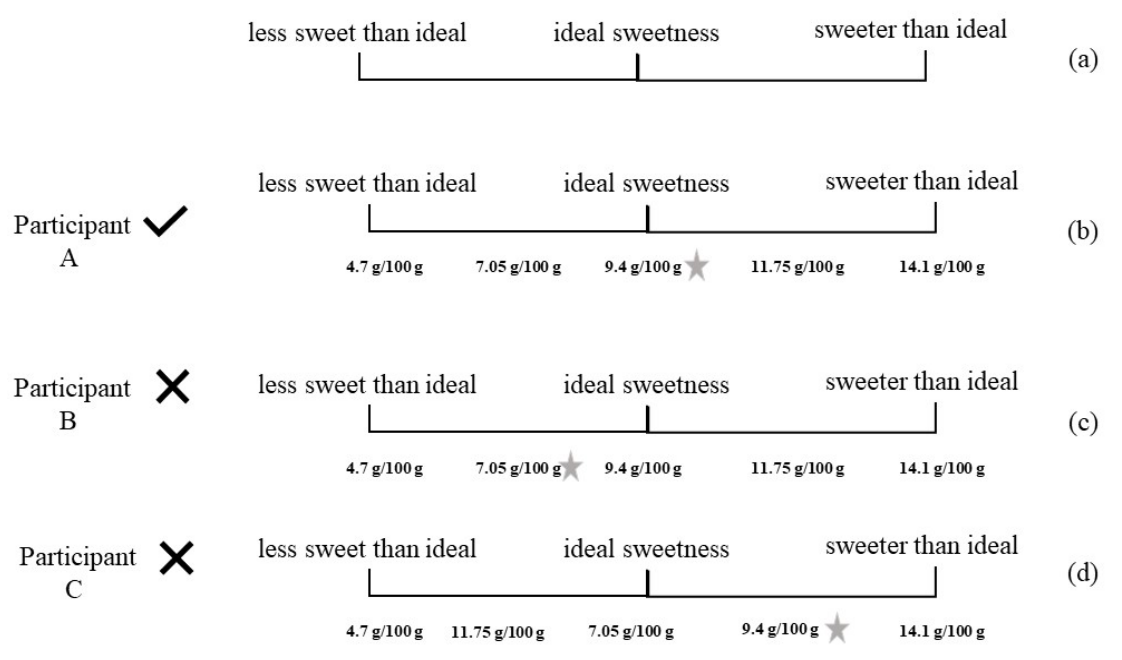

182

183 Figure 1: (a) Participant selection form. (b) Participant selection form from a selected

184 participant. (c) Participant selection form from a discarded participant. (d) Participant

185 selection form from a discarded participant. The star placed on the right side illustrates

186 the preferred sample. In the original form, the volunteer marked the sample number on

187 the scale. For didactic purposes, these codes were replaced with the sample concentration

188 in the figure.

189

190 Signal processing and CNN training

191 Figure 2 shows the network architecture that achieved the best performance. The best

192 number for $n$ was 20, as can be seen by the input layer shape. The other three fully

193 connected layers had 16, 16, and 64 neurons. Its dropout optimal value was 0.2 .

194 We used this optimal architecture to train the CNN with $70 \%$ of the data, while we

195 employed the remaining $30 \%$ for the test. 


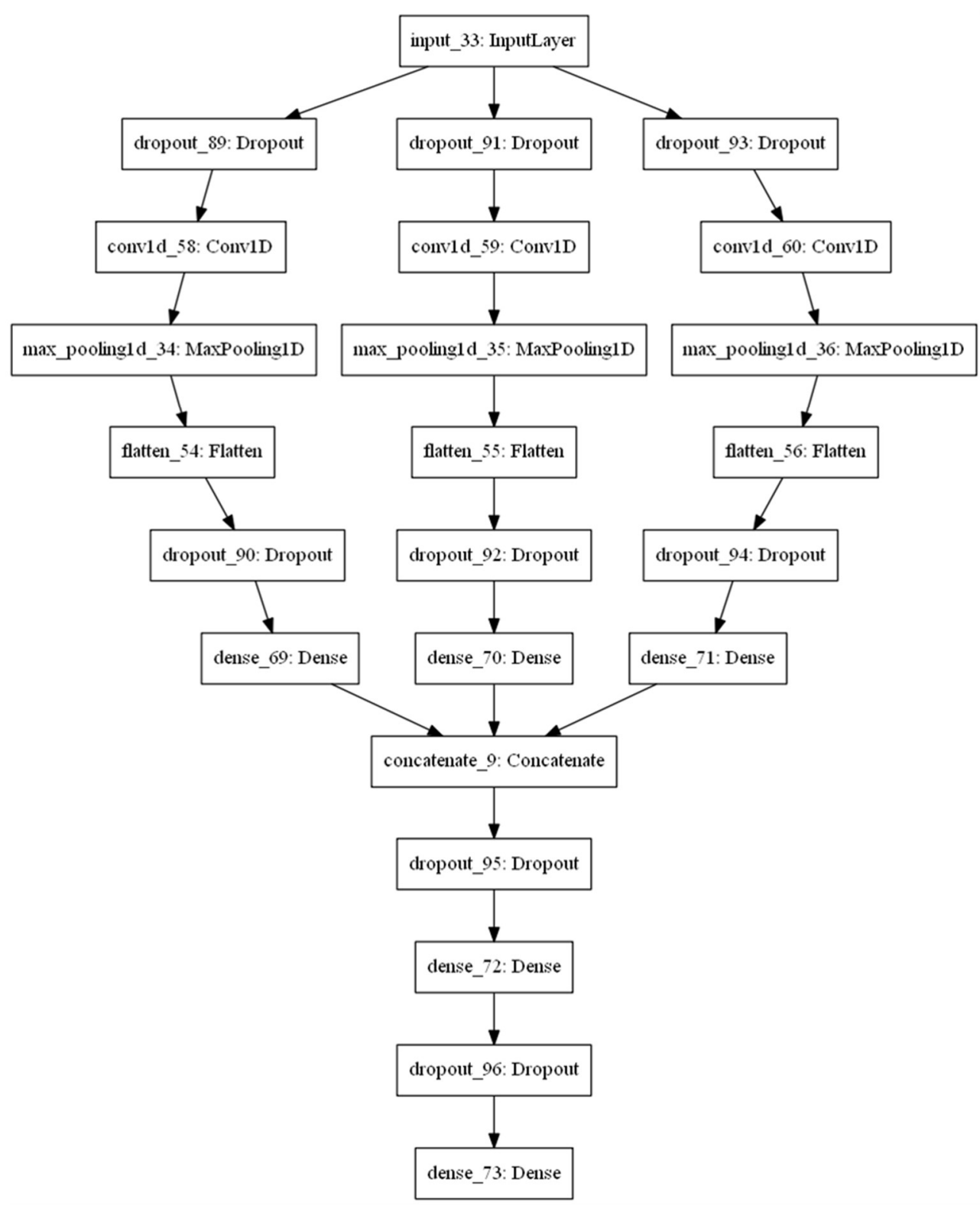

197 Figure 2: The convolutional neural network $(\mathrm{CNN})$ architecture that achieved the best

198 performance.

199

200 For visualization, Figure 3 presents the confusion matrix for this dataset. In the

201 confusion matrix, the horizontal axis is the predicted label, and the vertical axis is the

202 true label. The elements on the diagonal represent the numbers of correctly classified

203 samples. 


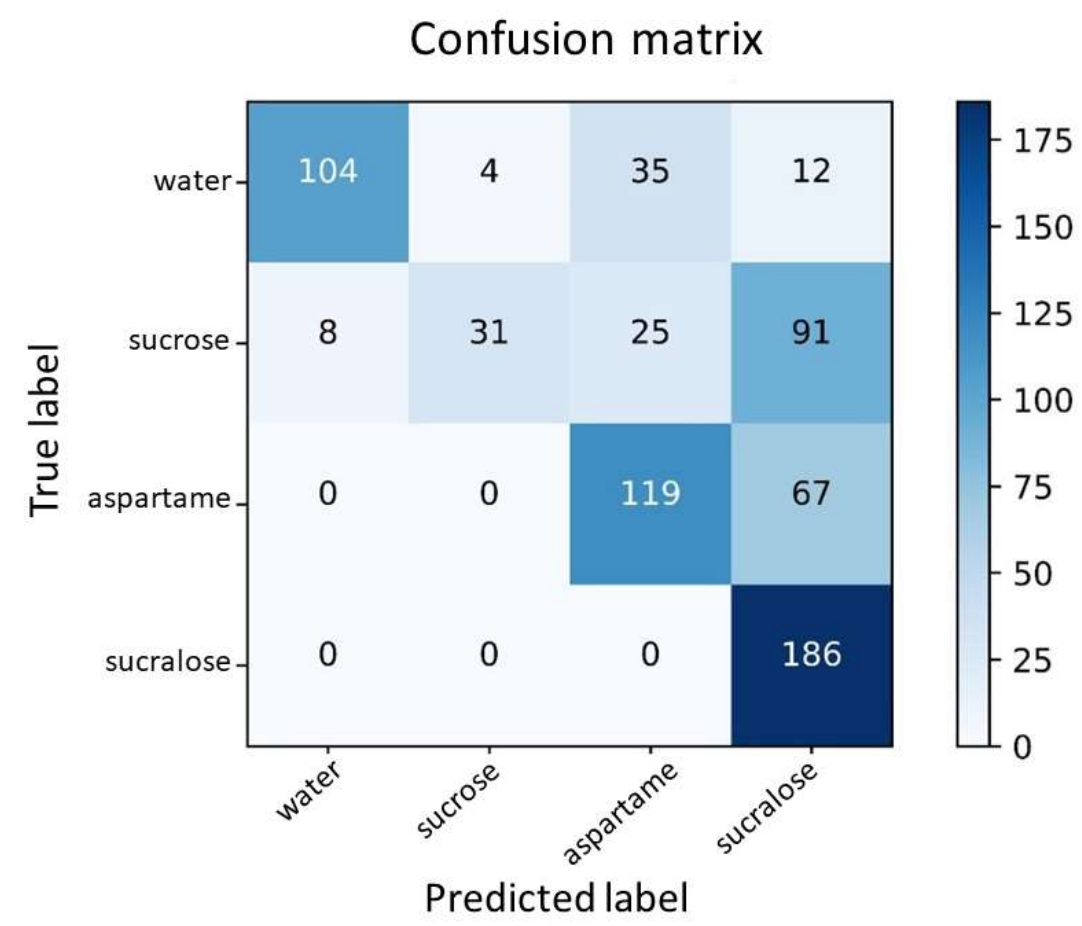

205 Figure 3: Confusion matrix for the classes water, sucrose, sucralose, and aspartame.

207 In Figure 3, for the water class, 104 samples were correctly predicted as water $(67.1 \%)$,

208 whereas 35 samples (7.7\%) were incorrectly predicted as aspartame and 4 samples

$209(2.6 \%)$ were incorrectly predicted as sucralose. For the sucrose class, 31 samples

$210(20.0 \%)$ were correctly predicted as sucrose, whilst 25 samples $(16.1 \%)$ were

211 incorrectly predicted as aspartame, and 91 samples $(58.7 \%)$ were incorrectly predicted

212 as sucralose. As for the aspartame class, 119 samples $(64.0 \%)$ were correctly classified,

213 while 67 samples $(36.0 \%)$ were incorrectly predicted as sucralose. Concerning the

214 sucralose class, 186 samples $(100.0 \%)$ were correctly classified. The main difficulty lay

215 in the classes of water and sucrose.

216 Table 1 lists the metrics results for the four stimuli (classes). As a result, the average

217 metrics were: 0.823 for accuracy, 0.750 for precision, 0.628 for recall and 0.611 for $f_{1}$

218 score. There are no studies using CNN in similar databases for comparison purposes, 
219 but these values are compatible with those obtained for studies involving brain signals

220 to assess emoticons (YIN ZHONG) and cerebral dominance (TORAMAN).

221 When we consider reference (water) only, the classification in Figure 3 indicated that

222 the average identification accuracy is $91,4 \%$ compared to an overall classification of

$22382.3 \%$. This work uses a drink instead of a solution and, in a first study of this nature, it

224 was important to be able to distinguish a critical reference well.

225

226 Table 1: Results of CNN classification performance for the four stimuli (classes).

227

\begin{tabular}{lcccc}
\hline & Accuracy & Precision & Recall & F $_{1 \text { score }}$ \\
\hline Water & 0,9135 & 0,9286 & 0,6710 & 0,7790 \\
Sucrose & 0,8123 & 0,8857 & 0,2000 & 0,3263 \\
Aspartame & 0,8138 & 0,6648 & 0,6398 & 0,6521 \\
Sucralose & 0,7507 & 0,5225 & 1,0000 & 0,6863 \\
\hline
\end{tabular}

228

229 When we compare general performance with reference performance, mainly $f_{1}$ score

230 that consider recall and precision measures simultaneously we can deduce that

231 misclassifications in sweeteners classes are greater that that in reference classe.

232 Analysing Figure 3 in more detail, suggests that the network is more sensitive to the

233 low-calorie sweetener classes than to the sucrose class. By evaluating the false positives

234 (FP) of the classification, aspartame can be predicted to be sucralose, but not sugar. In

235 turn, sucralose had no false positives. The most surprising result is that sucrose can be

236 classified both as sugar and low-calorie sweetener. In other words, evaluated low-

237 calorie sweeteners had not been confused with the caloric sweetener, but the caloric 
sweetener can be confused with the low-calorie sweetener. Andersen et al. (2019)

observed that similar tastes that are consciously indistinguishable can result in different

240 brain cortical activations. A similar result was obtained when gustatory evoked

241 potentials (GEPs) were used to assess the brain response to sucrose, aspartame, and

242 stevia in humans (Mouillot et al., 2020). The authors stated that, although sucrose, aspartame, and stevia led to the same taste perception, the GEPs showed that cerebral activation by these different sweet solutions had different recordings. They suggested that, besides the difference in taste receptors and cerebral areas activated by these

246 substances, neural plasticity and changes in the synaptic connections related to sweet

247 innate preference and sweet conditioning could explain the differences in cerebral

248 gustatory processing after sucrose and sweetener activation. The results presented

249 herein showed that this may be true.

250 Unlike Andersen et al. (2019) and Mouillot et al. (2020), in this study we evaluated a

251 beverage (i.e., passion fruit juice) with good acceptance after it is sweetened with

252 aspartame or sucralose (Rocha and Bolini, 2015b, 2015a) as a stimulus instead of

253 evaluating a sweet solution. This may explain FP rates in classification. During tasting,

254 the stimuli came not only from the sweet solution, but also from the other sensory

255 characteristics of the beverage.

256 Apart from using a beverage instead of a solution and from dispensing a taste delivery

257 system (Andersen et al., 2019; Frank et al., 2008; Jacquin-Piques et al., 2016;

258 Kobayakawa et al., 1996; Kobayakawa, 1999) that is common in this kind of

259 experimental design, we only placed two electrodes near the gustatory cortex. While

260 this is not a new strategy (Cincotti et al., 2002; Costa and Cabral, 2000; Hashida et al.,

261 2005), it is uncommon (Andersen et al., 2019; Frank et al., 2008; Jacquin-Piques et al., 
262 2016; Kobayakawa et al., 1996; Kobayakawa, 1999) and has advantages and

263 disadvantages. The main disadvantage is that it reduces the amount of data and makes

264 some feature extraction techniques unfeasible. On the other hand, developing

265 commercial applications based on a single EEG channel, such as the applications used

266 by Hashida et al. (2005) and Silva et al. (2005), is easy.

267 Andersen et al. (2019) observed that response discrimination by between-participant

268 qEEG is inferior to discrimination by within-participant qEEG. Therefore, we can

269 improve the results of this study by increasing the number of samples tested per

270 individual and also the number of individuals. This would allow us to train the network

271 for each participant, for instance. However, this approach would involve the use of a

272 taste delivery system to facilitate the increase in the number of samples.

273 This fact does not invalidate the main contribution of this study, which corroborates

274 recent studies (Andersen et al., 2019; Frank et al., 2008; Mouillot et al., 2020) stating

275 that similar stimuli, despite being consciously indistinguishable, may result in different

276 cortical responses. Moreover, this study included a beverage instead of a solution and

277 used only two monitoring positions (i.e., C3 and C4) over the gustatory cortex.

\section{Conclusions}

279 We have compared brain signals acquired in response to the consumption of passion

280 fruit juice sweetened with sucrose (caloric sweetener), sucralose, or aspartame (low-

281 calorie sweeteners). We used the artifact-free data to feed a CNN. The results indicated

282 that the brain responses can distinguish the juice sweetened with sucrose from the juice

283 sweetened with aspartame and sucralose.

284 Author's Contributions 
Conceptualization: Silva, A.C.S, Costa, E.J.X., Miñano, H.L.A. Data acquisition:

Writing - original draft: Silva, A.C.S. Writing -review \& aditing: Silva, A.C.S.,

Costa, E.J.X; Atzingen, G.V., Miñano, H.L.A. Funding acquisition: Silva, A.C.S.,

Project administration: Silva, A.C.S.

\section{Acknowledgments}

The authors thank Ajinomoto for the donation of aspartame (AminoSweet ${ }^{\mathrm{TM}}$ ).

\section{References}

Andersen, C. A.;Kring, M. L.;Andersen, R. H.;Larsen, O. N.;Kjaer, T. W.;Kidmose, U.;... Kidmose, P. 2019. EEG discrimination of perceptually similar tastes. Journal of Neuroscience Research, 97:241-252. https://doi.org/10.1002/jnr.24281

Bassoli, A.;Merlini, L. 2003. SWEETENERS | Intensive. In Encyclopedia of Food Sciences and Nutrition (pp. 5688-5695). Elsevier. https://doi.org/10.1016/B0-12227055-X/01172-X

Caballero, B. 2013. Sucrose: Dietary Sucrose and Disease. In Encyclopedia of Human Nutrition (pp. 231-233). Elsevier. https://doi.org/10.1016/B978-0-12-3750839.00257-9

Cincotti, F.;Mattia, D.;Babiloni, C.;Carducci, F.;Bianchi, L.;del R Millán, J.;... Babiloni, F. 2002. Classification of EEG mental patterns by using two scalp electrodes and Mahalanobis distance-based classifiers. Methods of Information in Medicine, 41:337-41. Retrieved from http://www.ncbi.nlm.nih.gov/pubmed/12425246

Costa, E. J. X.;Cabral, E. F. 2000. EEG-based discrimination between imagination of left and right hand movements using adaptive gaussian representation. Medical Engineering \& Physics, 22:345-348. https://doi.org/10.1016/S13504533(00)00051-5

\section{DELIZA, R.;MacFIE, H.;HEDDERLEY, D. 2005. THE CONSUMER SENSORY} PERCEPTION OF PASSION-FRUIT JUICE USING FREE-CHOICE PROFILING. Journal of Sensory Studies, 20:17-27. https://doi.org/10.1111/j.1745-459X.2005.050604.x

Frank, G. K. W.;Oberndorfer, T. A.;Simmons, A. N.;Paulus, M. P.;Fudge, J. L.;Yang, T. T.;Kaye, W. H. 2008. Sucrose activates human taste pathways differently from artificial sweetener. NeuroImage, 39:1559-1569. 
Gao, Z.;Zhang, K.;Dang, W.;Yang, Y.;Wang, Z.;Duan, H.;Chen, G. 2018. An adaptive optimal-Kernel time-frequency representation-based complex network method for characterizing fatigued behavior using the SSVEP-based BCI system. KnowledgeBased Systems, 152:163-171. https://doi.org/10.1016/J.KNOSYS.2018.04.013

Hashida, J. C.;Silva, A. C. de S.;Souto, S.;Costa, E. J. X. 2005. EEG pattern discrimination between salty and sweet taste using adaptive Gabor transform. Neurocomputing, 68:251-257. https://doi.org/10.1016/J.NEUCOM.2005.04.004

328

329

330

331

332

333

334

335

336

337

338

339

340

341

342

343

344

345

346

347

348

349

350

351

352

353

354

355

356

357

358

359

360

361

362

363

Hill, S. E.;Prokosch, M. L.;Morin, A.;Rodeheffer, C. D. 2014. The effect of non-caloric sweeteners on cognition, choice, and post-consumption satisfaction. Appetite, 83:82-88. https://doi.org/10.1016/j.appet.2014.08.003

Huang, A. L.;Chen, X.;Hoon, M. A.;Chandrashekar, J.;Guo, W.;Tränkner, D.;... Zuker, C. S. 2006. The cells and logic for mammalian sour taste detection. Nature, 442:934-938. https://doi.org/10.1038/nature05084

Jacquin-Piques, A.;Gaudillat, S.;Mouillot, T.;Gigot, V.;Meillon, S.;Leloup, C.;... Brondel, L. 2016. Prandial States Modify the Reactivity of the Gustatory Cortex Using Gustatory Evoked Potentials in Humans. Frontiers in Neuroscience, 9:. https://doi.org/10.3389/fnins.2015.00490

JASPER;H., H. 1958. The ten twenty electrode system of the international federation. Electroencephalography and Clinical Neurophysiology, 10:371-375. Retrieved from http://ci.nii.ac.jp/naid/10020218106/en/

Kobayakawa, T. 1999. Spatio-temporal Analysis of Cortical Activity Evoked by Gustatory Stimulation in Humans. Chemical Senses, 24:201-209. https://doi.org/10.1093/chemse/24.2.201

Kobayakawa, T.;Endo, H.;Ayabe-Kanamura, S.;Kumagai, T.;Yamaguchi, Y.;Kikuchi, Y.;... Ogawa, H. 1996. The primary gustatory area in human cerebral cortex studied by magnetoencephalography. Neuroscience Letters, 212:155-158. https://doi.org/10.1016/0304-3940(96)12798-1

Linforth, R. S. 2000. Developments in instrumental techniques for food flavour evaluation: future prospects. Journal of the Science of Food and Agriculture, 80:2044-2048. https://doi.org/10.1002/1097-0010(200011)80:14<2044::AIDJSFA753>3.0.CO;2-Z

Mouillot, T.;Parise, A.;Greco, C.;Barthet, S.;Brindisi, M.-C.;Penicaud, L.; .. JacquinPiques, A. 2020. Differential Cerebral Gustatory Responses to Sucrose, Aspartame, and Stevia Using Gustatory Evoked Potentials in Humans. Nutrients, 12:322. https://doi.org/10.3390/nu12020322

NABORS, L. O. 2002. Sweet Choices: Sugar Replacements for Foods and Beverages. Food Technology, 56:28-35. Retrieved from https://www.ift.org/news-andpublications/food-technology-magazine/issues/2002/july/features/sweet-choicessugar-replacements-for-foods-and-beverages

Naim, M.;Nir, S.;Spielman, A. I.;Noble, A. C.;Peri, I.;Rodin, S.;Samuelov-Zubare, M. 2002. Hypothesis of Receptor-Dependent and Receptor-Independent Mechanisms for Bitter and Sweet Taste Transduction: Implications for Slow Taste Onset and Lingering Aftertaste (pp. 2-17). https://doi.org/10.1021/bk-2002-0825.ch001 
Ohla, K.;Busch, N. A.;Lundström, J. N. 2012. Time for Taste-A Review of the Early Cerebral Processing of Gustatory Perception. Chemosensory Perception, 5:87-99. https://doi.org/10.1007/s12078-011-9106-4

Okamoto, M.;Dan, I. 2007. Functional near-infrared spectroscopy for human brain mapping of taste-related cognitive functions. Journal of Bioscience and Bioengineering, 103:207-215. https://doi.org/10.1263/jbb.103.207

PINHEIRO, M. V. S.;OLIVEIRA, M. N.;PENNA, A. L. B.;TAMIME, A. Y. 2005. The effect of different sweeteners in low-calorie yogurts - a review. International Journal of Dairy Technology, 58:193-199. https://doi.org/10.1111/j.14710307.2005.00228.x

Rocha, I. F. de O.;Bolini, H. M. A. 2015.a. Different sweeteners in passion fruit juice: Ideal and equivalent sweetness. LWT - Food Science and Technology, 62:861867. https://doi.org/10.1016/J.LWT.2014.10.055

Rocha, I. F. de O.;Bolini, H. M. A. 2015.b. Passion fruit juice with different sweeteners: sensory profile by descriptive analysis and acceptance. Food Science \& Nutrition, 3:129-139. https://doi.org/10.1002/fsn3.195

Silva, A. C. de S.;Arce, A. I. C.;Souto, S.;Costa, E. J. X. 2005. A wireless floating base sensor network for physiological responses of livestock. Computers and Electronics in Agriculture, 49:246-254. https://doi.org/10.1016/J.COMPAG.2005.05.004

Zorn, S.;Alcaire, F.;Vidal, L.;Giménez, A.;Ares, G. 2014. Application of multiple-sip temporal dominance of sensations to the evaluation of sweeteners. Food Quality and Preference, 36:135-143. https://doi.org/10.1016/j.foodqual.2014.04.003 